\section{Patient 2}

A 48-year-old man was referred to Yamaguchi University hospital for surgical treatment of a superior mediastinal tumor. A chest CT scan showed that the tumor was located mainly adjacent to the apical thoracic vertebra, but partially above the thoracic inlet (Figure 2, C). Magnetic resonance imaging showed low intensity on the T1-weighted sequence and high intensity on the T2-weighted sequence, which mimicked schwannoma. We attempted to perform totally thoracoscopic resection of the tumor. Careful manipulation was necessary for dissecting the tumor from sympathetic nerve and brachial plexus in the thoracic inlet. We dissected the tumor from the nerves carefully using scissors and an ultrasonic scalpel. The operation was completed totally thoracoscopically with no complications. The operation time was $122 \mathrm{~min}-$ utes, and intraoperative blood loss was $150 \mathrm{~mL}$. The patient was discharged on postoperative day 3. Pathologic examination revealed that the tumor was a schwannoma of the brachial plexus. A CT scan performed 5 months after the operation showed no evidence of recurrence of the tumor.

\section{DISCUSSION}

Resection of an extended superior mediastinal tumor is fraught with technical difficulties because of the anatomic complexity of the superior mediastinum and the thoracic inlet, where there is a large concentration of vital vessels and nerves. Moreover, it is difficult to identify the anatomic detail of the thoracic inlet, even via conventional thoracotomy because it is the farthest and narrowest area to reach; thus, the vagal nerve, recurrent nerve, and sympathetic nerve can be injured during tumor dissection. To avoid these risks, we used a high-definition video system (IMAGE HD, Storz, Germany) with a 30-degree angled scope. This system enabled us to visualize magnified and high-resolution images of individual structures around the thoracic inlet, from the best angles. We also used an ultrasonic scalpel to dissect the tumor without injuring the vital nerves or vessels, because it causes less heat damage than electrocautery to the adjacent structures or organs, ${ }^{3}$ and the narrow blade is useful for dissecting and cutting the structures safely in such a narrow space during thoracoscopic surgery. Apart from these instrumental uses, our strategy is useful for positioning the access ports. Because the thoracoscope and assistant instruments are inserted from the opposite side of the primary surgeon, they rarely interrupt the devices used by the primary surgeon (Figure 1, B).

\section{CONCLUSIONS}

These advantages allow the surgeon to carry out comfortable and less stressful manipulation than that required for conventional thoracotomy for extended superior mediastinal tumors.

\section{References}

1. Shigemura N, Akashi A, Nakagiri T, Matsuda H. VATS with a supraclavicular window for huge substernal goiter: an alternative technique for preventing recurrent laryngeal nerve injury. Thorac Cardiovasc Surg. 2005; 53:231-3.

2. Isowa N, Kikuchi R, Kunimoto Y, Ito K, Tokuyasu H, Fukuda K, et al. Successful resection of posterior mediastinal thyroid cancer by partial sternotomy combined with video-assisted thoracoscopy. Ann Thorac Cardiovasc Surg. 2007;13: 47-9.

3. Takahashi S, Fukuda I, Kuga T, Tanaka M. Exposure of the coronary artery using ultrasonic scalpel. J Thorac Cardiovasc Surg. 2003;125:1533-4.

\title{
A novel technique for identification of the lung intersegmental plane using dye injection into the segmental pulmonary artery
}

\author{
Seiichiro Sugimoto, MD, Takahiro Oto, MD, Kentaroh Miyoshi, MD, and Shinichiro Miyoshi, MD, \\ Okayama, Japan
}

\footnotetext{
From the Department of Cancer and Thoracic Surgery, Okayama University Graduate School of Medicine, Dentistry and Pharmaceutical Sciences, Okayama, Japan. Disclosures: Authors have nothing to disclose with regard to commercial support. Received for publication June 17, 2010; revisions received Aug 1, 2010; accepted for publication Sept 10, 2010; available ahead of print Oct 29, 2010.

Address for reprints: Seiichiro Sugimoto, MD, Department of Cancer and Thoracic Surgery, Okayama University Graduate School of Medicine, Dentistry and Pharmaceutical Sciences, 2-5-1 Shikata Cho, Okayama 700-8558, Japan (E-mail: sugimo-s@cc.okayama-u.ac.jp).

J Thorac Cardiovasc Surg 2011;141:1325-7

$0022-5223 / \$ 36.00$

Copyright (c) 2011 by The American Association for Thoracic Surgery doi:10.1016/j.jtcvs.2010.09.029
}

The diagnostic frequency of small-sized lung cancers has increased with the development and evolution of computed tomography. For small-sized lung cancers, lung segmentectomy is not inferior to lobectomy in selected patients. ${ }^{1,2}$ During lung segmentectomy, the intersegmental line, the 2-dimensional segmental border on the lung surface, is usually identified by inflation/deflation of the target lung segment. ${ }^{3}$ Because regions adjacent to the target lung segment can become inflated through collateral ventilation, it is difficult to identify the intersegmental line in 

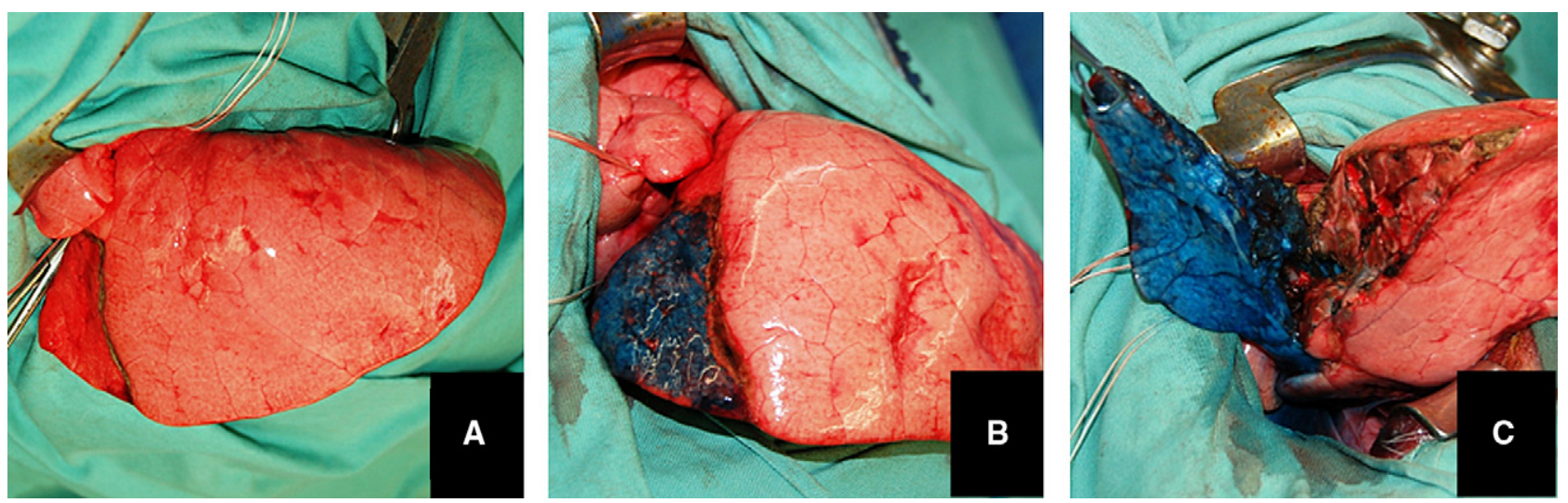

FIGURE 1. A, Intersegmental line identified through the bronchus (B7+8). B, Target segment (S7+8) stained through the pulmonary artery (A7+8). C, Intersegmental plane visualized in segmentectomy.

emphysematous lungs that show abundant collateral ventilation. Misaki and colleagues ${ }^{4}$ recently reported an alternative method for identification of the intersegmental line: infrared thoracoscopy after intravenous indocyanine green injection. This method is dependent on blood flow and is also suitable for emphysematous lungs; however, infrared thoracoscopy is not yet available worldwide, and it only allows identification of the intersegmental line and not the intersegmental plane, the 3-dimensional segmental border in the cutting plane. Although accurate identification of the intersegmental plane during lung segmentectomy can decrease air leakage from the dissected lung parenchyma and shorten hospital stay, no effective technique is currently available for identification and visualization of the intersegmental plane. As a novel technique for identifying the lung intersegmental plane, we applied direct injection of Indigo Carmine (Daiichi Sankyo, Tokyo, Japan) into the target segmental vessel, as for liver segmentectomy. ${ }^{5}$ The purpose of this study was to develop a novel technique for identification of the lung intersegmental plane that would also be suitable for emphysematous lungs.

\section{EXPERIMENTAL SUMMARY}

The experiments described herein were performed in 6 Landrace pigs weighing 25 to $30 \mathrm{~kg}$. The Animal Studies Committee at Okayama University approved all animal procedures. The right lung of the pig consists of the cranial, middle, caudal, and accessory lobes, and the left lung consists of the cranial and caudal lobes. Because of the anatomic difficulty of the right cranial lobar bronchus arising directly from the trachea, the left lung was used for this experiment. The pulmonary segmental bronchi of the pig are defined in accordance with those in human subjects: the first dorsal branch in the left cranial lobe corresponds to the apicoposterior segmental bronchus (B1+2); in the left caudal lobe the first dorsal branch corresponds to the superior seg- mental bronchus (B6), and the first lateral branch corresponds to the anteromedial basal segmental bronchus (B7 +8 ). The segments (S) and pulmonary arteries (PAs) are named after each bronchus. Three animals were examined for the $\mathrm{S} 1+2$ and $\mathrm{S} 6$ models, and 3 were examined for the $\mathrm{S} 7+8$ models.

To compare the techniques for identifying the lung segment through the PA and through the bronchus, first, the target segmental bronchus was clamped through a left thoracotomy after induction of anesthesia and endotracheal intubation. After identification of the deflated segmental area, the transition zone between the target segmental area and adjacent areas was marked to the visceral pleura by using electrocautery (Figure 1,A), and the target segmental bronchus was unclamped. Then the target segmental PA was ligated, and $10 \mathrm{~mL}$ (40 mg) of Indigo Carmine was injected into the PA distal to the ligation. The target segmental area showed blue staining, whereas the adjacent areas remained unstained (Figure 1, B). In all 9 target segments the intersegmental line identified by the dye injection was completely consistent with that identified by means of deflation, suggesting that identification through both the bronchus and the PA can delineate the intersegmental line accurately. We did not detect collateral circulation in these healthy animals that might cause the dye migration to the adjacent segments. We then proceeded with segmentectomy of one segment each in all 3 models. In all 3 models the target segmental area remained blue, without the color fading, during the intersegmental dissection, and the intersegmental plane was clearly visualized (Figure 1,C). Staplers were not used to visualize the intersegmental plane. Furthermore, the draining pulmonary vein from the target segment was also stained and identified without difficulty.

To examine the dye effect histologically, in the remaining 6 of the 9 segments, India ink was injected into the same site of the segmental PA as that of the initial injection of Indigo 


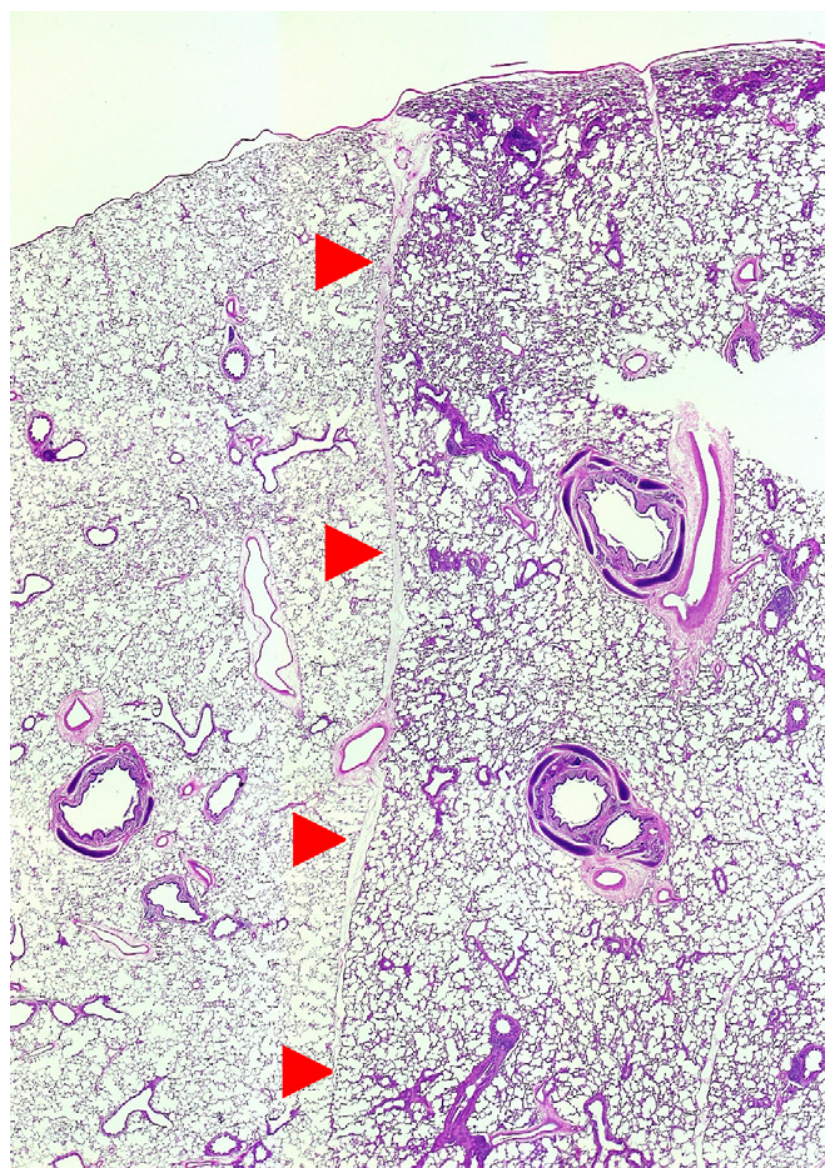

FIGURE 2. Microscopic intersegmental plane of S6 (arrows).
Carmine, dyeing the target area black. After left pneumonectomy, the lung specimens were fixed with formalin, embedded in paraffin, and stained with hematoxylin and eosin. The intersegmental plane was clearly visualized with the dye on microscopic examination (Figure 2), supporting our hypothesis that the intersegmental plane can be easily identified by means of dye injection.

\section{DISCUSSION}

The staining might affect the diagnosis of visceral pleural invasion in the clinical setting; however, it is expected to allow for better understanding of the intersegmental structure and facilitate intersegmental dissection during lung segmentectomy.

\section{References}

1. Keenan RJ, Landreneau RJ, Maley RH Jr, Singh D, Macherey R, Bartley S, et al. Segmental resection spares pulmonary function in patients with stage I lung cancer. Ann Thorac Surg. 2004;78:228-33.

2. Okada M, Koike T, Higashiyama M, Yamato Y, Kodama K, Tsubota N. Radical sublobar resection for small-sized non-small cell lung cancer: a multicenter study. J Thorac Cardiovasc Surg. 2006;132:769-75.

3. Okada M, Mimura T, Ikegaki J, Katoh H, Itoh H, Tsubota N. A novel videoassisted anatomic segmentectomy technique: selective segmental inflation via bronchofiberoptic jet followed by cautery cutting. J Thorac Cardiovasc Surg. 2007; 133:753-8.

4. Misaki N, Chang SS, Gotoh M, Yamamoto Y, Satoh K, Yokomise H. A nove method for determining adjacent lung segments with infrared thoracoscopy. J Thorac Cardiovasc Surg. 2009;138:613-8.

5. Makuuchi M, Hasegawa H, Yamazaki S. Ultrasonically guided subsegmentectomy. Surg Gynecol Obstet. 1985;161:346-50. 